\title{
sfcount: Command for count-data stochastic frontiers and underreported and overreported counts
}

DOI:

$10.1177 / 1536867 X 20953566$

Link to publication record in Manchester Research Explorer

\section{Citation for published version (APA):}

$\mathrm{Fe}, \mathrm{E} .$, \& Hofler, R. (2020). sfcount: Command for count-data stochastic frontiers and underreported and overreported counts. The Stata Journal, 20(3), 532-547. https://doi.org/10.1177/1536867X20953566

\section{Published in:}

The Stata Journal

\section{Citing this paper}

Please note that where the full-text provided on Manchester Research Explorer is the Author Accepted Manuscript or Proof version this may differ from the final Published version. If citing, it is advised that you check and use the publisher's definitive version.

\section{General rights}

Copyright and moral rights for the publications made accessible in the Research Explorer are retained by the authors and/or other copyright owners and it is a condition of accessing publications that users recognise and abide by the legal requirements associated with these rights.

\section{Takedown policy}

If you believe that this document breaches copyright please refer to the University of Manchester's Takedown Procedures [http://man.ac.uk/04Y6Bo] or contact uml.scholarlycommunications@manchester.ac.uk providing relevant details, so we can investigate your claim.

\section{OPEN ACCESS}


The Stata Journal (yyyy)

$v v$, Number $i i$, pp. $1-15$

\title{
sfcount: Command for Count Data Stochastic Frontiers and Underreported and Overreported Counts.
}

\author{
Eduardo Fé \\ University of Manchester \\ Manchester, U.K. \\ eduardo.fe@manchester.ac.uk
}

\author{
Richard Hofler \\ University of Central Florida \\ Orlando, FL, U.S.A. \\ richard.hofler@ucf.edu
}

\begin{abstract}
We introduce a new command, sfcount, to estimate Count Data Stochastic Frontier models. Although originally designed to estimate production and production-cost functions, this new command can be used to estimate mean regression functions when count data are suspected to be under/overreported.
\end{abstract}

Keywords: st0001, sfcount, stochastic frontier, underreporting, overreporting, low discrepancy series, Maximum Simulated Likelihood.

\section{Introduction.}

In this article we introduce a new Stata command, sfcount, which estimates the parameters of the Count Data Stochastic Frontier (CDSF) model in Fé and Hofler (2013).

Stochastic frontier models (Aigner et al. 1977, Meeusen and van den Broeck 1977) are central to the identification of inefficiencies in the production (and production costs) of continuously distributed outputs. In labour, industrial and health economics, production frontiers have also been adopted to explain deviations from maximal or minimal levels of non-tangible and/or non-pecuniary outcomes. However in these latter domains outcomes are often measured as counts (for example, the number of patents obtained by a firm or the number of infant deaths in a region). Although these latter fields of inquiry have not emphasised the idea of inefficiency in the 'production' of non-tangible and/or non-pecuniary outcomes, recent contributions (e.g. Fé|2013: Fé and Hofler 2013) suggest that inefficiencies are also present in these domains.

The need for specific count data models for stochastic frontiers arises because, not only this results in more efficient estimation (e.g. Greene 2004), but, more critically, inefficiency is typically not nonparametrically identified from data alone. Therefore researchers have to make specific assumptions regarding the distribution of inefficiency in the sample or the population. These assumptions define the class of admissible distribution underlying outcomes. Standard continuous data models attribute any negative (positive) skewness in the sample to inefficiencies in the production of economic goods (bads). The distributions of discrete outcomes are, however, typically skewed even in the absence of inefficiencies (e.g. the Poisson distribution) and the sign of skewness is generally independent of whether one is studying an economic good or an economic bad. 
sfcount

As a result, standard stochastic frontier models can fail to detect any inefficiency in production when the outcome of interest is a count -even when the underlying inefficiency is substantial.

The core count data stochastic frontier model is based on a mixed Poisson distribution with a log-halfnormal mixing parameter (or a Poisson log-halfnormal, PHN, in the parlance of Fé and Hofler 2013). Although the motivation behind this model was the estimation of stochastic frontiers under discrete valued outcomes, the PHN can be employed for modelling underreported counts as well as overreported counts. These situations are pervasive when studying worker's absenteeism (Winkelmann 1996), consumer data (Fader and Hardie 2000), drug abuse (Brookoff et al. 1993) or traffic accidents (Alsop and Langley 2001) among others. Among the models traditionally used for modelling these events the Beta-Binomial and Poisson-Lognormal models have been widely used. The PHN is a complement to these specifications.

The code presented here extends the catalogue of Stata commands pertaining to the stochastic frontier literature including the original Stata commands frontier and xtfrontier as the recent extensions sfcross and sfpanel by Belotti 2013). The original model in Fé and Hofler (2013) was cross-sectional in nature. Therefore, this code does not take into account individual time-invariant heterogeneity. In the continuous outcome stochastic frontier literature, panel data extensions abound. For an excellent review -including extensions- see Greene (2005); a recent important methodological contribution is Belotti and Ilardi (2018). Similarly, the original model in Fé and Hofler (2013) did not deal with endogenous regressors. This is an active area of research in the general stochastic frontier literature. Seminal contributions include Kutlu (2010), Griffiths and Hajargasht (2016) and Amsler et al. (2016) The development of a count data model with covariates endogenous to inefficiency is an unexplored area of work.

\section{Methods}

To introduce the PHN model we adopt the stochastic frontier terminology in Fé and Hofler (2013). The relationship to under/overreported counts models will be apparent from the context. There is a sample of $i=1, \ldots, n$ units containing data on a discrete outcome of interest $y_{i} \in\{0,1,2, \ldots\}$. The mean production frontier of $y$ is determined by the mapping

$$
\log \tilde{\lambda}=h(x ; \beta) .
$$

where $\tilde{\lambda} \in \mathbb{R}^{+}$. Conditional on a level of inefficiency (or level of under-/over- reporting) $\varepsilon \in \mathbb{R}^{+}$, the mean deterministic frontier is

$$
\log \lambda=h(x ; \beta) \pm \varepsilon
$$

Since we are modelling non-negative count data, we transform the last equation to

$$
\lambda=\exp (h(x ; \beta) \pm \varepsilon) .
$$

1. Another recent contributions are Karakaplan and Kutlu 2017b and Karakaplan and Kutlu (2017a). For accompanying code see Karakaplan (2017) 
Following convention, we assume that $y$, has an Poisson distribution conditional on a set of regressors, $x$, and $\varepsilon$, with $\lambda$ as the conditional mean of the distribution. The unconditional distribution follows by endowing $\varepsilon$ with an specific density. Following the convention in the Stochastic Frontier literature, Fé and Hofler (2013) assume that $\varepsilon$ follows a half normal distribution, so that

$$
f(\varepsilon)=f(\varepsilon ; \sigma)=\frac{2}{\sigma \sqrt{2 \pi}} \exp \left(-\frac{\varepsilon^{2}}{2 \sigma^{2}}\right) \mathbb{I}_{[0, \infty)} \text { for } \sigma_{\varepsilon}>0 .
$$

This density has the advantage of allowing some flexibility, thanks to its scale parameter. It also leads to a model whose first order moments are well defined (a property that is not shared by some popular distribution $\$^{2}$.

If $f(\varepsilon)$ is half normal we can write $\varepsilon=|u|$, where $u$ has a normal distribution. With this notation, and letting $h(x ; \beta)=x^{\prime} \beta$, the conditional distribution of $y$ given $x$ follows by averaging $\mathbb{P}(y \mid x, u)$ over the range of $u$,

$$
\begin{aligned}
\mathbb{P}(y \mid x ; \sigma, \beta) & =E\left[\frac{\left(\exp \left(-\exp \left(x^{\prime} \beta \pm \sigma|u|\right)\right)\right) \exp \left(y\left(x^{\prime} \beta \pm \sigma|u|\right)\right)}{y !}\right] \\
& =E\left[\text { Poisson }\left(\exp \left(x^{\prime} \beta \pm \sigma|u|\right)\right)\right]
\end{aligned}
$$

where expectations are taken with respect to the standard normal distribution. Fé and Hofler 2013 provide expressions for the moments of $f(\varepsilon)$ when this follows a half normal density function, as well as expressions for the conditional mean and variance of $y$. The PHN distribution does not have a closed form expression, however the integral in (2) can be approximated by simulation. Specifically, Fé and Hofler (2013) advocate combining Maximum Simulated Likelihood estimation of the PHN model with Halton sequences (Gentle 2003). Applying simulation, the conditional distribution of $y_{i}($ for $i=1, \ldots, n)$ is approximated by the sum:

$$
\mathbb{P}(y \mid x ; \theta) \approx \hat{\mathbb{P}}\left(y \mid x ; s_{h}, \theta\right)=\frac{1}{H} \sum_{h=1}^{H} \operatorname{Poisson}\left(\exp \left(x^{\prime} \beta \pm \sigma\left|s_{h}\right|\right)\right)
$$

where $s_{h}$ are the terms of a, possibly randomized, Halton sequence. The infeasible loglikelihood $L_{n}=\sum_{i=1}^{n} \log \mathbb{P}(y \mid x ; \theta)$ can be approximated by $L_{n, h}=\sum_{i=1}^{n} \log \hat{\mathbb{P}}\left(y \mid x ; s_{h}, \theta\right)$. The analytical derivatives of $L_{n, h}$ are given by

$$
\frac{\partial L_{n, h}}{\partial \theta}=\sum_{i=1}^{n} \frac{1}{\hat{\mathbb{P}}\left(y_{i} \mid x_{i} ; s_{h}, \theta ;\right)} \frac{1}{H} \sum_{h=1}^{H} \operatorname{Poisson}\left(\tilde{\lambda}_{i, h}\right)\left(y_{i}-\tilde{\lambda}_{i, h}\right)\left\{\begin{array}{c}
x_{i} \\
\pm\left|s_{h}\right|
\end{array}\right\}
$$

where $\tilde{\lambda}_{i, h}=\exp \left(x_{i}^{\prime} \beta \pm \sigma\left|s_{h}\right|\right)$. The value $\hat{\theta}_{M S L}$ making the above system of equations equal to zero is the Maximum Simulated Likelihood estimator of $\theta$. When the PHN is a correct representation of the underlying data generating process, $\hat{\theta}_{M S L}$ is a consistent, asymptotically normal and efficient estimator of the true parameter value, as

2. For example, when that $\varepsilon$ has gamma distribution with parameters $\alpha>0$ and $\delta>0$ such that $\delta=\alpha$; see Fé and Hofler 2013 
follows from properties 1 and 2 above. Standard errors can be computed via the BHHH estimator or the minus inverse of the Hessian Matrix of $L_{n, h}$.

Tests of hypotheses can rely on the Wald-Score-LR trinity. Testing the null hypothesis of no inefficiency is of particular interest. The null hypothesis would be

$$
H_{0}: \sigma=0
$$

in which case PHN collapses to a standard Poisson model. A formal test can be computed via a likelihood ratio comparing the resulting value with the quantiles of a $\chi_{1}^{2}$ distribution.

\subsection{Estimating cross-sectional inefficiency.}

Although the parameters of the frontier are of interest in themselves, the ultimate goal of most stochastic frontier analyses is to obtain approximate efficiency scores (i.e. measures of the deviations away from either maximal or minimal values of the outcome) for each individual in the sample. Following Jondrow et al. (1982) cross-sectional inefficiency scores, $v=\exp ( \pm|u|)$, can be estimated via $E(v \mid y, x)$. Using Bayes' theorem

$$
f(v \mid x, y)=\frac{\mathbb{P}(y \mid x, v) f(v)}{\mathbb{P}(y \mid x)}
$$

so that

$$
E(v \mid y, x)=\int v f(v \mid x, y) d v .
$$

The latter expression does not have a closed form. However, we may still approximate the relevant integral via simulation. The simulated $E(v \mid y, x)$ for the parametric Mixed Poisson model is

$$
\hat{v}_{i}=E\left(v_{i} \mid x_{i}, y_{i}\right) \approx \frac{\sum_{h=1}^{H} \exp \left( \pm\left|s_{h}\right| \sigma\right) \operatorname{Poisson}\left(\exp \left(x_{i}^{\prime} \beta+\sigma\left|s_{h}\right|\right)\right)}{\sum_{h=1}^{H} \operatorname{Poisson}\left(\exp \left(x_{i}^{\prime} \beta+\sigma\left|s_{n}\right|\right)\right)}
$$

Two remarks are important here. First, the distributions of $v$ and $\hat{v}$ are not the same $3^{3}$ and the lower and upper tails of the distribution of $v$ will be misreported. From a stochastic frontier perspective, this means that $\hat{v}$ penalizes outstanding firms and rewards the least efficient individuals -although the average efficiency in the sample is correctly approximated. However, the estimator is unbiased in the unconditional sense $E(\hat{v}-v)=0($ Wang and Schmidt 2009).

Second, in applications, the scores depend critically on the term $\operatorname{Poisson}\left(\exp \left(x_{i}^{\prime} \beta+\right.\right.$ $\left.\sigma\left|s_{h}\right|\right)$. If the mean of this distribution, $\exp \left(x_{i}^{\prime} \beta+\sigma\left|s_{h}\right|\right)$ is too large in relation to $\mathrm{Y}$ for any one observation, then Poisson $\left(\exp \left(x_{i}^{\prime} \beta+\sigma\left|s_{h}\right|\right)\right.$ will be approximately 0 . Therefore, for that observation the cross-sectional estimate of inefficiency will be $0 / 0$ which Stata reports as missing value. We thus recommend researchers to ensure that the explanatory variables are measured in meaningful units albeit of small magnitude.

3. As can be seen by noting that $\operatorname{var}(v)=\operatorname{var}(E(v \mid x, y))+E(\operatorname{var}(v \mid x, y))$, (and hence, $\hat{v}$ has smaller variance) 


\title{
3 Stata syntax
}

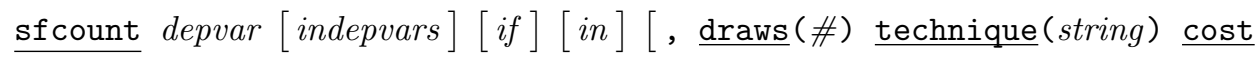 \\ cluster (vcetype) ]
}

where depvar is the dependent variable and indepvars are the explanatory variables.

\subsection{Options.}

draws (\#) Specify the number of Halton draws (the default is 200). The model is estimated via Maximum Simulated Likelihood. To approximate the likelihood function of the Poisson-log-Half-Normal model, the command uses Halton sequences (a low-discrepancy sequence). Halton sequences ensure a good coverage of the unit interval (e.g. Niederreiter 1992).

technique (string) specifies the optimization technique. The default option is the modified Newton-Raphson (nr). You can switch among dfp (Davidon-FletcherPowell), bhhh (Berndt-Hall-Hall-Hausman) and bfgs (Broyden-Fletcher-GoldfarbShanno).

cost Specifies that the underlying model is a cost function (or, equivalently, an overreporting or deviation above the minimal level function). By default sfcount estimates a production function (or equivalently, an underreporting or deviation below the maximal level function).

cluster (string) specifies the name of a variable that creates intra-group correlation, relaxing the usual requirement that the observations be independent.

\subsection{Cross-sectional estimates of inefficiency.}

The command automatically generates a variable named inefficiency collecting the cross sectional scores.

\subsection{Example 1. Stochastic Frontier.}

For this example, we generated 1000 observations from a PHN distribution with mean $\exp \left(1+x_{1}+x_{2}-v\right)$ where $x_{j} \sim$ Uniform $[0,1]$ and $v$ has a halfnormal distribution with $\sigma=1$ (the code to generate the data appears in the discussion of the Monte Carlo simulation below). This yielded the following output

\begin{tabular}{|c|c|c|c|c|c|c|c|}
\hline & & & & Number of & obs & $=$ & 1,000 \\
\hline dep & Coef. & Std. Err. & z & $P>|z|$ & {$[95 \%$} & Conf. & Interval] \\
\hline \multicolumn{8}{|l|}{ eq1 } \\
\hline $\mathrm{x} 1$ & 1.009668 & .070637 & 14.29 & 0.000 & .871 & 2215 & 1.148114 \\
\hline
\end{tabular}


sfcount

\begin{tabular}{rr|rrrrrr} 
& x2 & .9658712 & .0731504 & 13.20 & 0.000 & .822499 & 1.109243 \\
& _cons & 1.024507 & .0665457 & 15.40 & 0.000 & .8940796 & 1.154934 \\
\hline eq2 & & & & & & & \\
& & & & & & & \\
& _cons & -.0217397 & .051502 & -0.42 & 0.673 & -.1226818 & .0792023 \\
\hline
\end{tabular}

Note: _cons in eq2 corresponds to the log of the standard deviation

of the mixing log-half-normal parameter

Ho: Inefficiency not present in the sample

$\operatorname{chi2}(1)=\quad 325.51$

Prob $>$ chi2 $=\quad 0.00$

The output follows standard Stata convention. The first block of results, eq1, presents the estimates of the structural coefficients of the regressors (including an intercept). The second block of results, eq2, presents the estimate of $\log (\sigma)$. Therefore, the point estimate of $\sigma$ can be retrieved by using the transformation $\sigma=\exp ($ _cons) in eq2. Below the table of main results one finds the likelihood ratio test for $H_{0}: \sigma=0$. In this case, the statistic equals 325.51 with associated p-value of 0.00 (and thus one would reject the null hypothesis).

The sf count command automatically calculates the cross-sectional inefficiency scores and stores those into a new variable, inefficiency. The following summary statistics compare the average and actual estimated inefficiencies.

\begin{tabular}{r|rrrrr} 
Variable & Obs & Mean & Std. Dev & Min & Max \\
\hline expv & 1,000 & .5210644 & .2519012 & .0130767 & .9992961 \\
inefficiency & 1,000 & .5297692 & .1732635 & .1411413 & .9017366
\end{tabular}

The variable expv collects the true inefficiency scores, whereas the second variable, inefficiency, collects the estimated inefficiency scores. It is clear that, as pointed out by Wang and Schmidt (2009), the cross-sectional estimator is unbiased in an unconditional sense and thus it provides very accurate estimates of the average inefficiency. Even though figure 1 reveals a discrepancy between the actual and the estimated scores (which is expected since, in this simulation, $x_{j}$ do not provide any information about the inefficiency parameter) there is also a strong positive correlation between the actual and estimated inefficiencies. The smaller standard deviation in the estimated inefficiencies is due to $\hat{v}$ begin a shrinkage of $v$ towards its mean (as noted by Wang and Schmidt 2009).

\section{Monte Carlo Simulation.}

To verify the properties of the method and the robustness of the software, we run a Monte Carlo simulation. We drew samples of 100 observations from a PHN model with conditional mean $\exp \left(1+x_{1}+x_{2}-\sigma v\right)$ where $x_{j} \sim$ Uniform $[0,1]$ and $v$ has a halfnormal distribution with $\sigma \in\{0.5,1,2\}$. We estimated the parameters of this model 500 times. We wrote a short ado file, mc1, to generate the data, estimate the model and return the 


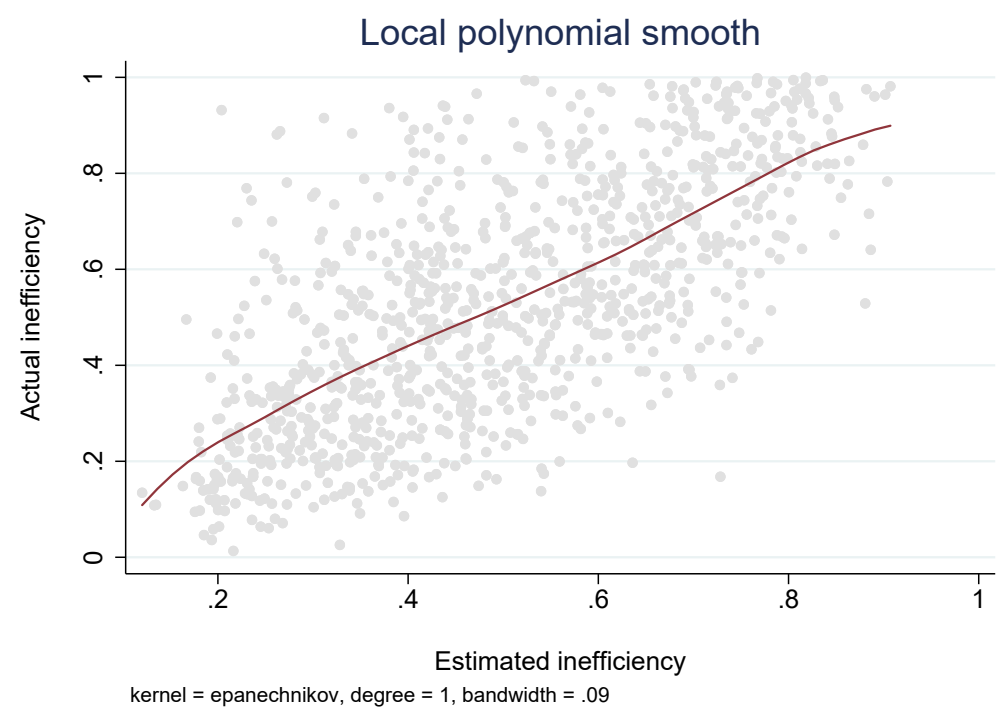

Figure 1: Real vs estimated cross-sectional inefficiency.

result to Stata's simulate command 4 . The results of each experiment are presented now. For $\sigma=0.5$, we obtained the following results.

\begin{tabular}{r|rrrrr} 
Variable & Obs & Mean & Std. Dev. & Min & Max \\
\hline b0 & 500 & .9968649 & .1848761 & .4428408 & 1.652993 \\
b1 & 500 & 1.00012 & .1827392 & .4863433 & 1.519381 \\
b2 & 500 & .9707445 & .1909647 & .4019189 & 1.443396 \\
s & 500 & .4656329 & .1557467 & .0001077 & .8336025 \\
reject & 500 & .654 & .4761696 & 0 & 1
\end{tabular}

Here b0 corresponds to the intercept, b1, b2 are the coefficients of $\mathrm{x} 1, \mathrm{x} 2$ respectively, $\mathbf{s}$ is the estimate of $\sigma$ and reject is the proportion of times the null hypothesis $\sigma=0$ was rejected by the likelihood ratio test. It is promptly observed that, even for the very small sample size considered, the parameters of the model are very accurately estimated on average. Specifically, the critical parameter $\sigma$ was tightly concentrated around the true value of 0.5 . The empirical power of the likelihood ratio test was $65 \%$ which is acceptable give the very small sample size considered. Similar conclusions were reached with the alternative specification $\varsigma^{5}$

What would occur if the standard normal-halfnormal model in Aigner et al. (1977) were fitted to these data instead? We illustrate the situation through a simulation for both the cost and production frontier cases. We maintain the same design but we will focus on the case $\sigma=1$ for simplicity. Following convention in Aigner et al. (1977), we

4. The code for the simulation can be obtained from the authors upon request.

5. The results can be also obtained from the authors upon request. 
fit the model

$$
\log y=\theta_{0}+\theta_{1} \log x_{1}+\theta_{2} \log x_{2} \pm u+v
$$

where now, $u$ is the inefficiency term (distributed halfnormal) and $v$ is the idiosyncratic, zero mean error term. The case $+u$ corresponds to the cost function, whereas the case $-u$ corresponds to the production frontier. The code to run this simulation is similar to mc1.ado, however sfcount is replaced by the built-in command frontier and the variables are transformed to logs. The critical parameters in this model are the standard errors of $u$ and $v$, say $\sigma_{u}, \sigma_{v}$. Specifically, the ratio $\lambda=\sigma_{u} / \sigma_{v}$ is the commonly used measure of the magnitude of inefficiency in the sample. In addition to this, the likelihood ratio test of $\sigma_{u}=0$ serves, as before, as the statistic to draw inferences regarding the statistical significance of inefficiency in the sample. The result of this is

\begin{tabular}{r|rrrrr} 
Variable & Obs & Mean & Std. Dev. & Min & Max \\
\hline b0 & 500 & .2211184 & .0890768 & .0012678 & .6122401 \\
b1 & 500 & .2313553 & .0854484 & .0486661 & .6074979 \\
b2 & 500 & 2.499497 & .2308907 & 1.412019 & 3.118477 \\
s & 500 & 2158507 & 8511800 & .0325458 & $5.28 e+07$ \\
reject & 500 & .726 & .4464556 & 0 & 1 \\
\hline k3 & 500 & -.1886938 & .1433905 & -.6482261 & .213362
\end{tabular}

The critical quantities in the simulation are $\mathrm{k} 3$ and $\mathbf{s}$ ( which corresponds to the ratio $\left.\lambda=\sigma_{u} / \sigma_{v}\right)$. The production stochastic frontier model expects negative skewness in the $\log$ of the dependent variable, and on this occasion, the average skewness happens to be negative (but this is not always, as illustrated below). Indeed, the type of skewness exhibited by $\log Y$ will depend on the specific parameterization of the generating process (note that the untransformed data will always have a positively skewed distribution). The most critical aspect of these results is that the parameter $\lambda$ is estimated very imprecisely. The large value of the estimated parameter would suggest that too often the model cannot separate inefficiency from pure noise, and that all the variation in the sample is erroneously attributed to inefficiency. In contrast, the likelihood ratio test only rejects the null hypothesis about $70 \%$ of the time. The result for the cost frontier are similarly worrying,

\begin{tabular}{r|rrrrr} 
Variable & Obs & Mean & Std. Dev. & Min & Max \\
\hline b0 & 498 & .2829813 & .0885495 & .0617461 & .6670547 \\
b1 & 498 & .2877539 & .0910561 & .0603849 & .6406236 \\
b2 & 498 & 2.79976 & .3151728 & 2.19097 & 3.677881 \\
s & 498 & 97321.05 & 1311344 & .0013252 & $2.15 e+07$ \\
reject & 498 & .3815261 & .4862496 & 0 & 1 \\
\hline k3 & 498 & .0985375 & .2989368 & -.7290995 & .9630092
\end{tabular}

In this case, maximum likelihood failed to converge in 2 replications. As with the production frontier case, the parameter $\lambda$ is imprecisely estimated (because all variation tends to be attributed to inefficiency). Yet, paradoxically, the significance of the inefficiency term is rejected only about $38 \%$ of the time. The critical aspect of this result is that although the distribution of $\log Y$ has the right type of skewness (positive), its 
magnitude is very small; therefore the cost stochastic frontier model fails to identify any inefficiency, even though this is prevalent in the data.

An even more problematic example arises when we let $\sigma=3$ and try to estimate a production frontier. Here the skewness of $\log Y$ is positive, which presents a violation of one of the assumptions underlying the continuous data stochastic frontier model. In this instance, the latter method fails to detect any inefficiency at all,

\begin{tabular}{r|rrrrr} 
Variable & Obs & Mean & Std. Dev & Min & Max \\
\hline b0 & 500 & .1504993 & .1210131 & -.2304306 & .8188418 \\
b1 & 500 & .1507706 & .1221521 & -.2453529 & .7454497 \\
b2 & 500 & 1.335596 & .4623144 & .6625592 & 3.285585 \\
s & 500 & 479674 & 3430413 & .0114056 & $3.44 e+07$ \\
reject & 500 & .056 & .230152 & 0 & 1 \\
\hline k3 & 500 & .2978432 & .2033263 & -.285708 & .9867306
\end{tabular}

As seen from the above results, the likelihood ratio test rejects the null hypothesis at the nominal $5 \%$ level, whereas $\lambda$ remains to be imprecisely estimated.

In summary, the continuous data stochastic frontier model can be problematic in practice when data are coming from the PHN specification.

\section{Example: The distribution of infant deaths in England.}

We next illustrate the use of the sfcount command in practice. Specifically, we model the conditional distribution of infant deaths in England during 2015 and 2016. The help file accompanying the sfcount command details how to reproduce the results of this exercise.

Infant deaths have a large opportunity cost for societies and constitute a marker of the overall health status of a population. Commonly cited risk factors are parental risk behaviour, pollution, economic deprivation and the quality of health providers, although a large proportion of infant deaths are not attributable to any specific cause (and are catalogued as Sudden Infant Death Syndrome). It is unclear, however, if the latter deaths still show systematic variation across different areas even after taking into account the effect of measurable determinants of infant deaths. The PHN can help us to detect which areas overreport infant deaths conditional on the area's characteristics (that is, which areas are inefficient in the production of infant deaths).

In order to illustrate the workings of the PHN when addressing this question, we downloaded data on infant deaths by local area for the years 2015 and 2016 from the website of the UK Office for National Statistics (ONS). We complemented these data with information on local areas characteristics from 2011 UK Census. The focus of the analysis in this exercise is socioeconomic status and air quality. Socioeconomic status has been shown to correlate with health and wealth. Air pollutants to the atmosphere can induce respiratory disease (including bronchitis, pneumonia, allergies or asthma). 


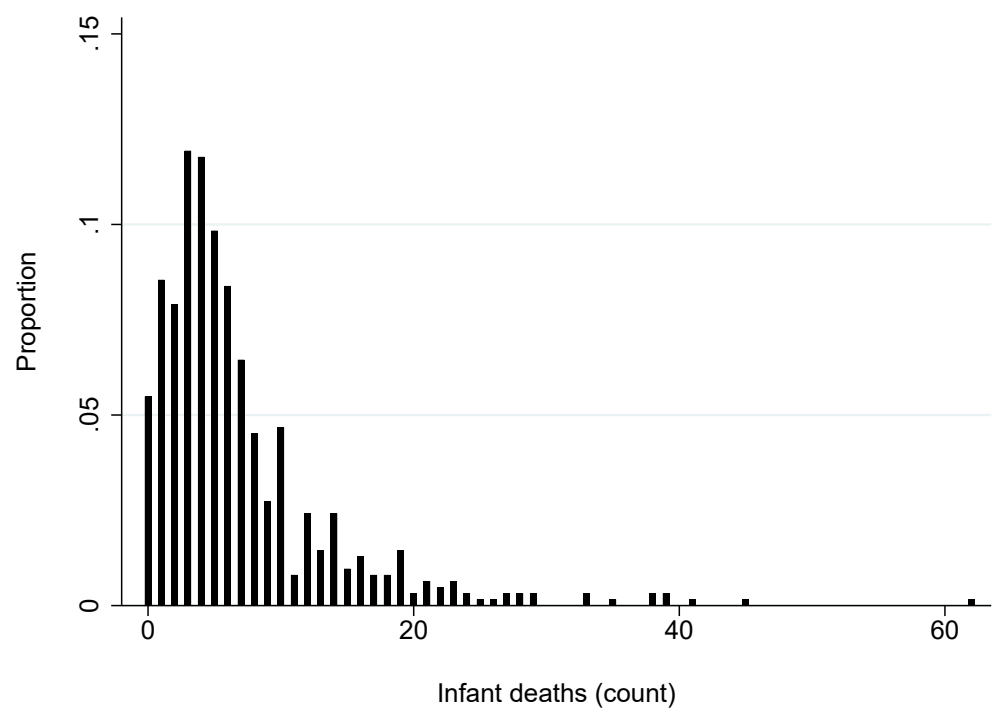

Figure 2: Distribution of infant deaths in England. Years 2015 and 2016.

Among these pollutants, nitrogen oxides ( by-products of fuel consumption and the production of electricity) are thought to be important determinants of respiratory diseases.

We proxy each area's socio-economic status with, first, the number of people claiming income benefits per 1000 of the population and, second, the area's employment rate. Low birth weight is a risk factor for infant mortality, therefore in our model we also incorporate the percentage of babies born at a gestational age of greater than or equal to 37 weeks and with a birth weight of less than 2,500g. Our indicator of air quality is the area's average nitrogen oxide emissions intensity score, $\mathrm{NO}_{x}$. This is a 8 point scale with higher scores indicating higher emissions. We found a few discrepancies between the names and geographic boundaries in the 2011 UK Census and the ONS' most recent data files containing the counts of infant deaths. Given the limited scope of this example, we opted to discard those areas for which data on the covariates was not available for that reason. This leaves us with full data for 309 local areas. For the purposes of this analysis, we pooled the 2015 and 2016 data, while areas' characteristics were imputed from the 2011 Census.

Descriptive statistics of our limited sample are provided in Table 1. The average number of deaths across England was 6.9, however the distribution is skewed, with a long right tail, as can be seen in Figure 2 (the maximum number of deaths observed was 62 , in the city of Manchester; note that London has been disaggregated in 31 sub areas). The average population in each area is 139,680 inhabitants, whereas the average proportion of underweight births is $7.1 \%$. On average, $12.9 \%$ of the population were claiming income benefits, whereas the average employment rate was $76 \%$. The average 


\begin{tabular}{lrrrr}
\hline \hline & Mean & S.D. & Min & Max \\
\cline { 2 - 5 } & & & & \\
Number of infant deaths & 6.945 & 7.054 & 0 & 62 \\
Nitrogen Oxide Emissions Score & 4.144 & 1.649 & 1.143 & 8 \\
\% Underweight births & 7.180 & 1.323 & 2.800 & 11.60 \\
Population & 139680.0 & 83175.8 & 24457 & 715402 \\
Employment rate & 76.82 & 3.992 & 64.73 & 84.45 \\
Year 2016 & 0.500 & 0.500 & 0 & 1 \\
East of England & 0.149 & 0.356 & 0 & 1 \\
East Midlands & 0.129 & 0.336 & 0 & 1 \\
London & 0.100 & 0.301 & 0 & 1 \\
North East & 0.0324 & 0.177 & 0 & 1 \\
North West & 0.120 & 0.325 & 0 & 1 \\
South East & 0.214 & 0.410 & 0 & 1 \\
South West & 0.107 & 0.309 & 0 & 1 \\
West Midlands & 0.0874 & 0.283 & 0 & 1 \\
Yorkshire and the Humber & 0.0615 & 0.240 & 0 & 1 \\
$N$ & & & & \\
$N$ & & 618 & & \\
\hline \hline
\end{tabular}

Table 1: Descriptive statistics.

$\mathrm{NO}_{x}$ score in the sample was 4.1. However, variation is vast, ranging from 1.1 to 8 .

Before undertaking our analysis, and in view of the results presented in the Monte Carlo section, we studied the empirical distribution of the logarithm of the count of infant deaths. This variable would be sitting at the core of any stochastic frontier analysis using the benchmark continuous data models (e.g. the normal-half normal model). The mean of this variable is based on 584 observations instead of 618 (5.5\% of the areas report 0 deaths), and equals 1.642. Importantly, its skewness equals -0.0444 . Parametric cost stochastic frontier models, however, expect the dependent variable to exhibit positive skewness. Indeed, the distribution of the log-deaths variable does not seem to be skewed at all, and a standard tests of normality based on the third and fourth moments of the variable did not reject the null hypothesis (sktest; p-value 0.5883). Therefore, data does not seem to support the premises of standard continuous output stochastic frontier models. This suggests that a standard continuous data frontier model will not provide a good fit for this variable, as already discussed in the Monte Carlo section. Unsurprisingly the normal-half-normal model struggled to converge to a solution (due to a 'not concave likelihood').

Having discarded the continuous data stochastic frontier model, we proceeded to estimate a battery of PHN models under nested conditioning sets. Table 2 presents the estimated coefficients of these models. As expected, population size is an important determinant of infant deaths, with larger populations seeing a higher number of deaths. 


\begin{tabular}{|c|c|c|c|c|}
\hline & $(1)$ & $(2)$ & 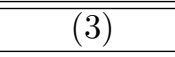 & (4) \\
\hline log Population & $\begin{array}{c}0.396^{* * *} \\
(6.16)\end{array}$ & $\begin{array}{c}0.357^{* * *} \\
(5.74)\end{array}$ & $\begin{array}{c}0.248^{* * *} \\
(4.30)\end{array}$ & $\begin{array}{c}0.268^{* * *} \\
(4.82)\end{array}$ \\
\hline$\%$ Underweight births & & $\begin{array}{l}0.0391 \\
(1.25)\end{array}$ & $\begin{array}{c}0.0111 \\
(0.41)\end{array}$ & $\begin{array}{c}-0.00691 \\
(-0.26)\end{array}$ \\
\hline Income benefit claimants (per 1000 pop.) & & $\begin{array}{c}-0.0281^{* * *} \\
(-3.44)\end{array}$ & $\begin{array}{c}-0.0178^{*} \\
(-2.42)\end{array}$ & $\begin{array}{c}-0.0185^{*} \\
(-2.56)\end{array}$ \\
\hline Employment rate & & $\begin{array}{c}-0.0968^{* * *} \\
(-13.00)\end{array}$ & $\begin{array}{c}-0.0582^{* * *} \\
(-7.77)\end{array}$ & $\begin{array}{c}-0.0656^{* * *} \\
(-7.96)\end{array}$ \\
\hline $\mathrm{NO}_{x}$ & & & $\begin{array}{c}0.245^{* * *} \\
(14.20)\end{array}$ & $\begin{array}{c}0.255^{* * *} \\
(11.80)\end{array}$ \\
\hline Year 2016 indicator & & & & $\begin{array}{c}0.0499 \\
(1.01)\end{array}$ \\
\hline Intercept & $\begin{array}{c}-3.901^{* * *} \\
(-5.14)\end{array}$ & $\begin{array}{c}4.245^{* * *} \\
(4.73)\end{array}$ & $\begin{array}{l}1.731^{*} \\
(2.11)\end{array}$ & $\begin{array}{l}2.092^{*} \\
(2.37)\end{array}$ \\
\hline Regional indicators & - & - & - & $\mathrm{Y}$ \\
\hline $\log \sigma$ & $\begin{array}{c}0.135^{* *} \\
(3.08)\end{array}$ & $\begin{array}{c}-0.0822 \\
(-1.82)\end{array}$ & $\begin{array}{c}-0.277^{* * *} \\
(-6.00)\end{array}$ & $\begin{array}{c}-0.377^{* * *} \\
(-7.12)\end{array}$ \\
\hline
\end{tabular}

Table 2: PHN model (conditional mean). $t$ statistics in parentheses. ${ }^{*} p<0.05,{ }^{* *}$ $p<0.01,{ }^{* * *} p<0.001$ 


\begin{tabular}{lcccc}
\hline \hline & & & & \\
& Mean & S.D. & Min. & Max. \\
\cline { 2 - 5 } & & & & \\
East of England & 1.937 & 0.763 & 1.152 & 5.479 \\
East Midlands & 1.849 & 0.533 & 1.278 & 3.552 \\
London & 1.913 & 0.672 & 1.113 & 3.699 \\
North East & 1.791 & 0.451 & 1.268 & 2.723 \\
North West & 1.921 & 0.838 & 1.149 & 6.461 \\
South East & 1.903 & 0.720 & 1.195 & 6.158 \\
South West & 1.870 & 0.596 & 1.300 & 4.320 \\
West Midlands & 1.753 & 0.427 & 1.255 & 3.132 \\
Yorkshire and the Humber & 2.302 & 1.516 & 1.256 & 6.227 \\
& & & & \\
Total & 1.908 & 0.755 & 1.113 & 6.461 \\
& & & & \\
\hline \hline
\end{tabular}

Table 3: Inefficiency by region

Importantly, we observe that socioeconomic status is negatively associated with infant deaths. Specifically, higher employment rates are strongly associated with lower count of deaths; however, we found that income benefits and infant deaths are negatively correlated. The significance of this contradictory result is, however, sensitive to the structure of the model, which casts some doubts about the reliability of this finding. We did not find any significant association between low birth weight and infant deaths. The most striking result, however, is the very strong association between air pollution and infant deaths. Specifically, higher levels of nitrogen oxides are associated with higher infant deaths.

All models considered return a statistically significant $\log \sigma$, which suggests that there is substantial 'inefficiency' in the sample, in the form of higher levels of deaths given the levels predicted by our model. Table 3 summarises the estimated inefficiency by each of the nine constituent areas. The average value of $\hat{\nu}$ for the whole sample is 1.9. There is substantial variation across regions. Inefficiency is lowest in the West Midlands and the North East (1.7). However, while the average estimated inefficiency score for most other regions sits at 1.8-1.9, the estimated inefficiency score for Yorkshire and the Humber is 2.3. Yorkshire and the Humber has the second largest employment rate in the sample, and the second lowest $\mathrm{NO}_{x}$ emissions score; however it has a relatively highest level of benefit claimants and underweight births. Given the prominence of employment rate and $\mathrm{NO}_{x}$ scores in our model, it would appear that Yorkshire is under-performing compared to other equally well off areas of England. In particular, this area seems to exhibit levels of infant death that more than double their predicted levels, given the area's socio-economic and environmental credentials.

We conclude this illustration of the sfcount command in practice with a number of 
caveats regarding the preceding application. A number of significant limitations restrict the scope and interpretation of the results. First, the model only captures association between the dependent and independent variables. In particular, we fall short of making any causal claims, specially in view that deaths, socioeconomic status and air quality might be either jointly determined or influenced by common unmeasurable factors. These factors might also determine the amount of inefficiency in the data. Second, we have considered a very limited number of explanatory variables and the latter were imputed from past observations. Thus, not only there is a considerable risk that our results are driven by latent heterogeneity, but measurement error is also likely to bias our results away from any causal parameter. Ultimately, however, the preceding analysis must be understood within the context of an illustration of our new command in practice.

\section{Conclusion.}

We have introduced a new command, sfcount, to estimate the Count Data Stochastic Frontier models in Fé and Hofler (2013). We have illustrated the implementation of this method and, through simulations, we have further illustrated the need for such a method. Although originally designed to estimate production and production-cost functions, our command can be used to estimate mean regression functions when count data are suspected to be under/overreported. The latter situations are common in empirical applications.

\section{References}

Aigner, D., C. Lovell, and P. Schmidt. 1977. Formulation and Estimation of Stochastic Frontier Production Function Models. Journal of Econometrics. 6: 21-37.

Alsop, J., and J. Langley. 2001. Under-reporting of motor vehicle traffic crash victims in New Zealand. Accident Analysis and Prevention 33(3): 353 - 359.

Amsler, C., A. Prokhorov, and P. Schmidt. 2016. Endogeneity in stochastic frontier models. Journal of Econometrics 190(2): 280 - 288. Endogeneity Problems in Econometrics.

Belotti, F. 2013. Stochastic frontier analysis using Stata. Stata Journal 13(4): 719 $758(40)$.

Belotti, F., and G. Ilardi. 2018. Consistent inference in fixed-effects stochastic frontier models. Journal of Econometrics 202(2): 161 - 177.

Brookoff, D., E. A. Campbell, and L. M. Shaw. 1993. The underreporting of cocainerelated trauma: drug abuse warning network reports vs hospital toxicology tests. American Journal of Public Health 83(3): 369-371.

Fader, P. S., and B. G. S. Hardie. 2000. A note on modelling underreported Poisson counts. Journal of Applied Statistics 27(8): 953-964. 
Fé, E. 2013. Estimating production frontiers and efficiency when output is a discretely distributed economic bad. Journal of Productivity Analysis 39(3): 285-302.

Fé, E., and R. Hofler. 2013. Count data stochastic frontier models, with an application to the patents-R\&D relationship. Journal of Productivity Analysis 39(3): 271-284.

Gentle, J. E. 2003. Random Number Generation and Monte Carlo Methods, 2nd Edition. Springer.

Greene, W. 2004. Econometric Analysis. Prentice Hall.

- 2005. Fixed and Random Effects in Stochastic Frontier Models. Journal of Productivity Analysis 23(1): 7-32.

Griffiths, W. E., and G. Hajargasht. 2016. Some models for stochastic frontiers with endogeneity. Journal of Econometrics 190(2): 341 - 348. Endogeneity Problems in Econometrics.

Jondrow, J., I. Materov, K. Lovell, and P. Schmidt. 1982. On the Estimation of Technical Inefficiency in the Stochastic Frontier Production Function Model. Journal of Econometrics 19: 233-238.

Karakaplan, M. 2017. Fitting endogenous stochastic frontier models in Stata. Stata Journal 17(1): 39-55.

Karakaplan, M. U., and L. Kutlu. 2017a. Handling endogeneity in stochastic frontier analysis. Economics Bulletin 37: 889-891.

—. 2017b. Endogeneity in panel stochastic frontier models: an application to the Japanese cotton spinning industry. Applied Economics 49(59): 5935-5939.

Kutlu, L. 2010. Battese-Coelli estimator with endogenous regressors. Economics Letters 109(2): $79-81$.

Meeusen, W., and J. van den Broeck. 1977. Efficiency Estimation from Cobb-Douglas Production Functions with Composed Error. International Economic Review 18: $435-444$.

Niederreiter, H. 1992. Random Number Generation and Quasi-Monte Carlo Methods. Society for Industrial and Applied Mathematics.

Wang, W., and P. Schmidt. 2009. On the distribution of estimated technical efficiency in stochastic frontier models. Journal of Econometrics 148: 36-45.

Winkelmann, R. 1996. Markov Chain Monte Carlo Analysis of Underreported Count Data with an Application to Worker Absenteeism. Empirical Economics 21: 575-587.

\section{About the authors}

Eduardo Fé is Senior Lecturer in Social Statistics at the University of Manchester.

Richard Hofler is Professor of Economics at the University of Central Florida. 